REVISTA PROYECCIONES $\mathrm{N}^{\circ}$ 7: 97-119

JUNIO 1984 - I.S.S.N. 0716-0917

\title{
UN JUEGO BAYESIANO CON VALOR DE INFORMACIÓN
}

ARNOLDO PRADO C. $(*)$

\section{INTRODUCCION.}

En ciertas situaciones reales que pueden modelarse según un jue go bipersonal, el nivel de información de cada jugador respecto a los pa rámetros puede no ser total, incluso puede ocurrir que el comportamiento racional de cada jugador esté sujeto al nivel del conocimiento que tenga cada uno respecto al nivel de conocimiento de su contrincante. Estas consideraciones y una metodología de análisis apropiada fueron formuladas por Harsanyi (1967) y (1968), quien propuso un modelo de juego que denomina juego Bayesiano que permite a cada jugador, frente a situaciones de incertidumbre, estructurar un método conducente a reglas de deci sión óptimas en una perspectiva bayesiana.

En el trabajo que se expone se recurre a las ideas de Harsanyi introduciendo además los conceptos de caracterización de cada jugador, el de confiabilidad y el del valor de información. La idea de la carac

(*) Departamento de Matemáticas, Facultad de Ciencias, Universidad del Norte. 
terización se identifica con el concepto de información que un jugador tiene sobre la modalidad de juego de su opositor. La confiabilidad se identifica con la distribución de probabilidad que cada uno asigna a las diversas caracterizaciones de su contrario y el valor de información como la variación del pago esperado cuando un jugador se aparte del criterio del punto de equilibrio.

\section{DEFINICIONES BASICAS.}

Sea $G^{*}=\left(S_{1}, S_{2} ; U_{1}, U_{2}\right)$ un juego bipersonal bimatricial donde para $i=1,2, S_{i}$ es el conjunto de estrategias puras del juga dor i $\mathrm{y} \mathrm{U}_{i}$ su respectiva función de pago. Entonces $G$ se reformula en los términos:

$$
\mathrm{G}=\left\{\mathrm{S}_{1}, \mathrm{~S}_{2} ; \mathrm{V}_{1}, \mathrm{~V}_{2} ; \mathrm{C}_{1}, \mathrm{C}_{2} ; \mathrm{P}_{1}, \mathrm{P}_{1}^{\prime} ; \mathrm{P}_{2}, \mathrm{P}_{2}^{\prime} ; \mathrm{R}_{1}, \mathrm{R}_{2}\right\}
$$

Para diferenciar ambos juegos, $G^{*}$ se denomina "juego convencional" y G "juego bayesiano equivalente".

En este modelo, las componentes del juego G tienen los siguien tes significados:

$S_{i}=$ conjunto finito de estrategias puras del jugador $i(i=1,2)$

$v_{i}=$ función de pago esperado por el jugador $i$.

$c_{i}=\left\{c_{i}\right\}=$ conjunto de características del jugador $i$.

$R_{1}$ y $R_{2}$ = funciones de distribuciones condicionales que, en opinión del jugador analista, asigna el jugador $i$ sobre el espacio $\mathrm{C}_{3-i}$ de su contrincante. Así:

$$
R_{1}=R_{1}\left(c_{2} \mid c_{1}\right) y R_{2}=R_{2}\left(c_{1} \mid c_{2}\right) .
$$




\subsection{Justificación de las hipótesis básicas.}

Ciertamente, las nuevas hipótesis suministran nuevas bases de análisis, con tal que tengan tanto una clara significación intuitiva como posibilidades de concretarse en situaciones del mundo real. Efectiva mente, ambos requisitos pueden ser alcanzados en este caso, como se seña la a continuación, en los órdenes que se indican:

La caracterización de cada jugador, ya sea como "conservador" - "agresivo", comprende al tipo de criterio de optimalidad que emplee ca da jugador. Así $c_{i}=c_{i}^{1}$ caracteriza a $i$ como conservador si $i$ adopta el criterio del punto de equilibrio y $c_{i}=c_{i}^{2}$ lo caracteriza como agresivo. En forma extensiva el conjunto $C$, será:

$$
c=\left\{\left(c_{1}^{1}, c_{2}^{1}\right) ;\left(c_{1}^{1}, c_{2}^{2}\right) ;\left(c_{1}^{2} c_{2}^{1}\right) ;\left(c_{1}^{2}, c_{2}^{2}\right)\right\}
$$

Así pues, en términos intuitivos, pueden darse las cuatro siguientes alternativas:

1) Jugador "conservador" versus jugador "conservador"

2) "Conservador" versus "agresivo".

3) "Agresivo" versus "conservador".

4) "Agresivo" versus "Agresivo".

Como consecuencia de lo anterior, los pagos esperados dependen del comportamiento de cada jugador y así, las funciones de pago asumirán cuatro valoraciones distintas, según los espacios donde cada jugador escoja, en opinión del analista, sus estrategias mixtas.

La existencia de una función de distribución objetiva $R^{*}$ es una consecuencia de la postulación de la existencia de las funciones de distribución condicionales. Solo es posible concebir una distribución condicional cuando se supone la existencia de alguna distribución conjunta que la genera. Su origen puede encontrarse en la experiencia de cada jụ 
gador analista, o bien que sea simplemente un dato derivado de un estudio previo, fuera del contexto de esta forma especifica de juego. Por ejemplo, no es aventurado suponer que el enfrentamiento entre dos duopolistas en un proceso de competencia de mercado, sea un proceso aleatorio en el cual la caracterización de cada uno como "conservador" o "agresivo" siga una ley de distribución accesible para cualquier analista y resulta do de todo un historial de esta situación.

Objetivamente, se sabe que el jugador 1 asigna una probabilidad a cada caracterización de su contrario. Sabe que la probabilidad, 1lama da en este caso confiabilidad, que el jugador 2 sea agresivo, es decir que el vector $c_{2}$ asuma el valor $c_{2}^{2}$, es $r_{1}(2)$. En forma análoga, objeti vamente el jugador 2 tiene una confiabilidad $r_{2}(1)$ que el jugador 1 es agresivo o equivalentemente, la probabilidad que el vector $\mathrm{c}_{1}$ asuma el valor $c_{1}^{2}$ es, en opinión del jugador $2, r_{2}(1)$.

Así pues, en términos del jugador 1, que analiza el juego sobre bases objetivas, la función de distribución $R^{*}$, definida sobre el conjun to $C=C_{1} \times C_{2}$, debe satisfacer las siguientes relaciones:

$$
\begin{aligned}
& \left.1^{\circ}\right) R^{*}\left(c_{2}=c_{2}^{2}\right)=r_{1}(2)<1 \\
& \left.2^{\circ}\right) R^{*}\left(c_{1}=c_{1}^{2}\right)=t_{2}(1)<1
\end{aligned}
$$

$r_{1}$ (2) $\mathrm{y}_{2}$ (1) tienen un matiz que las diferencia. Conceptualmente, el primer valor se refiere a la conjetura personal de 1 sobre lo que hará 2 en términos de su caracterización como agresivo, mientras que $t_{2}(1)$, es una conjetura que formula 1 como si él fuera el jugador 2 sobre la carac terización de 1 como agresivo. Ciertamente, ambos valores tienen un carácter personal, pero heurístico.

Ahora bien, desde un punto de vista enteramente convencional, el analista puede argumentar que la caracterización que él supone de su contrincante, no está de modo algunos condicionada a la postura que él adopte. Ciertamente, esta es una hipótesis razonable y está avalada 
precisamente, por la incertidumbre sobre lo que realmente va a ocurrir.

Así pues, si las variables $c_{1} y_{2} c_{2}$ son independientes estocásti camente, entonces se tendrá:

$$
\begin{aligned}
R^{*}\left(c_{2}\right) & =R^{*}\left(c_{2}, c_{1}^{1}\right)+R^{*}\left(c_{2}, c_{1}^{2}\right) \\
& =R^{*}\left(c_{2} \mid c_{1}\right) .
\end{aligned}
$$

En particular:

1) $R^{*}\left(c_{2}=c_{2}^{2}\right)=R *\left(c_{2}^{2} \mid c_{1}^{1}\right)=R *\left(c_{2}^{2} \mid c_{1}^{2}\right)$

$$
=r_{1}(2)
$$

2) $R^{*}\left(c_{2}=c_{2}^{1}\right)=R^{*}\left(c_{2}^{1} \mid c_{1}^{1}\right)=R^{*}\left(c_{2}^{1} \mid c_{1}^{2}\right)$

$$
=1-r_{1}(2)
$$

3) $R^{*}\left(c_{1}=c_{1}^{1}\right)=R *\left(c_{1}^{1} \mid c_{2}^{1}\right)=R *\left(c_{1}^{1} \mid c_{2}^{2}\right)$

$$
=t_{2}(1)
$$

4) $R^{*}\left(c_{1}=c_{1}^{2}\right)=R^{*}\left(c_{1}^{2} \mid c_{2}^{1}\right)=R^{*}\left(c_{1}^{2} \mid c_{2}^{2}\right)$

$$
=1-t_{2}(1)
$$

Es decir, en opinión del jugador analista, en este caso el jugador 1 , la probabilidad de que el jugador 2 sea agresivo dado $c_{1}$, es $r_{1}(2)$, pe ro evidentemente, de acuerdo a la definición de las distribuciones con dicionales $R_{i}$, esta probabilidad, es precisamente $R_{1}\left(c_{2}=c_{2}^{2} \mid c_{1}\right)$.

Análogamente, resulta de las relaciones 3) que $R^{*}\left(c_{1}=c_{1}^{2} \mid c_{2}\right)$ es equivalente a $R_{2}\left(c_{1}=c_{2}^{2} \mid c_{2}\right)$. 


\section{FORMULACION DEL MODELO BAYESIANO.}

De acuerdo a la teminología de los párrafos anteriores, el mode lo bayesiano equivalente para el juego planteado en el párrafo 3.2 , tiene la siguiente estructura:

$$
\mathrm{G}^{*}=\left\{\mathrm{S}_{1}, \mathrm{~S}_{2} ; \mathrm{C}_{1}, \mathrm{C}_{2} ; \mathrm{P}_{1}, \mathrm{P}_{2}, \mathrm{P}_{2}^{\prime} ; \mathrm{V}_{1}, \mathrm{~V}_{2} ; \mathrm{R}^{*}\right\}
$$

Estrictamente, el juego $G^{*}$ tiene como ámbito de incertidumbre las funciones $v_{1}, v_{2}$ de las cuales el analista, en este caso el jugador 1 , conoce totalmente sus estructuras y sus valoraciones, al tomar c todos sus valores sobre $\mathrm{C}=\mathrm{C}_{1} \times \mathrm{C}_{2}$. Naturalmente, las estrategias puras que figuran en cada caso, son aquellas que pueden ser elegidas con probabili dad positiva por las distribuciones correspondientes a los espacios que caracterizan cada uno de los valores del vector

$$
c=\left(c_{1}, c_{2}\right) \in C=c_{1} \times c_{2} \text {. }
$$

Ciertamente, el modelo así planteado, difiere del modelo bayesiano con información completa, en un hecho substancial, ahora el conocimiento de los pagos posibles para cada jugador es objetivo y, por 10 tanto, común a todas las opiniones que los distintos analistas del juego pudieran formular.

En definitiva, las alternativas posibles de pagos es un dato del problema.

2.1. Estrategias normalizadas y forma normal.

De acuerdo a la definición general de estrategias normalizadas, éstas, en el caso que se está anlizando, son funciones con dominio el conjunto

$$
c_{i}=\left\{c_{i}^{1}, c_{i}^{2}\right\}
$$


y con valores en el correspondiente espacio de estrategias puras $\mathrm{S}_{i}$.

Así pues, el espacio de estrategias normalizadas del jugador 1 , será simplemente identificado con el espacio producto $\mathrm{S}_{1} \mathrm{x}_{1}$, esto es $\left(S_{1}\right)=S_{1} \times S_{1}$. Análogamente, el espacio de estrategias normalizadas del jugador 2 será $\left(\mathrm{S}_{2}\right)=\mathrm{S}_{2} \mathrm{x}_{2}$. El número de elementos en $\left(\mathrm{S}_{1}\right)$ será $\mathrm{K}_{1}^{2}$ y el de $\left(\mathrm{S}_{2}\right)$ será $\mathrm{K}_{2}^{2}$. Los elementos de $\left(\mathrm{S}_{i}\right)$ se anotan

$$
\left(\mathrm{s}_{i}{ }^{\mathrm{i}}\right) ; i=1,2 ; \quad k_{i}=1,2,3, \ldots, \mathrm{k}_{i}^{2}
$$

Las estrategias mixtas del juego, en su forma original, las cua les eran elementos de $\mathrm{P}_{i} \circ$ de $\mathrm{C}\left(\mathrm{P}_{i}\right)$, en la forma normal quedarán formalmente estructuradas por las siguientes consideraciones:

1) El espacio $P_{i}$ es un simplex euclideano de dimensión $K_{i}{ }^{-1}$, es decir, los elementos de $\mathrm{P}_{i}$ son $\mathrm{K}_{1}$-uplas de números no negativos cuya suma es 1.

En símbolos:

$$
\begin{gathered}
\mathrm{p}_{i} \in \mathrm{p}_{i} \Longrightarrow \mathrm{p}_{i}=\left(\mathrm{p}_{i}^{1}, \mathrm{p}_{i}^{2}, \ldots, \mathrm{p}_{i}^{\mathrm{k}}\right) \\
\qquad \sum_{i}^{\mathrm{k}_{i}=1} \mathrm{p}_{i}^{\mathrm{k}}=1 \\
\left(\forall \mathrm{k}_{i} \in \mathrm{H}_{i}\right) \quad\left(\mathrm{p}_{i}{ }^{\mathrm{k}} \geqslant 0\right) ; \mathrm{H}_{i}=\left(1, \ldots, \mathrm{k}_{i}\right)
\end{gathered}
$$

2) El espacio $C\left(P_{i}^{\prime}\right)$ es el espacio cuyos elementos son $K_{i}^{\prime}-1$ uplas, $K_{i}^{\prime}<K_{i}$, de elementos no negativos cuya suma es uno. Así:

$$
\begin{aligned}
& p_{i}^{\prime} \in c\left(p_{i}^{\prime}\right) \Longrightarrow p_{i}^{\prime}=\left(p_{i}^{\prime} p_{i}^{2}, \ldots p_{i}^{k^{\prime}}\right) \\
& \left(\forall k_{i}^{\prime} \in H_{i}^{\prime}\right) \quad\left(p_{i}^{k_{i}^{\prime}} \geqslant 0\right) ; H_{i}^{\prime}=\left(1, \ldots, K_{i}^{\prime}\right)
\end{aligned}
$$




$$
\begin{aligned}
& \qquad \sum_{k_{i}^{\prime}=1}^{k_{i}^{\prime}} p_{i}^{k_{i}^{\prime}}=1 \\
& \text { Además }\left(\forall k_{i}^{\prime} \in H_{i}^{\prime}\right)\left(\exists p_{i}^{\prime} \in C\left(P_{i}^{\prime}\right)\right)\left(p_{i}{ }^{k}\left(s_{i}{ }^{\prime}\right)>0\right)
\end{aligned}
$$

El significado de esta exigencia no es más que una expresión de la elección que hace el contrincante de i del conjunto donde escogerá sus estrategias mixtas, o equivalentemente del conjunto de estrategias puras, a las cuales prefiere asignar a priori, probabilidades positivas.

3) Del punto 1) se deduce que $\mathrm{si} \mathrm{p}_{i} \mathrm{y}_{i}$ son dos distribuciones de pro babilidad sobre $\mathrm{s}_{i}$ entonces $\mathrm{p}_{i} \circ \mathrm{q}_{i}$ es una distribución de probabili dad sobre $\mathrm{S}_{i} \times \mathrm{S}_{i}$, en que $\mathrm{p}_{i} \mathrm{Oq}_{i}$ es ciertamente, la medida de probabili dad producto entre $p_{i}$ y $q_{i}$. Así pues:

$$
p_{i}, q_{i} \in P_{i} \Longrightarrow p_{i} \circ q_{i} \in P_{i} o p_{i}=P_{i}^{2}
$$

- equivalentemente el espacio de estratecrias mixtas para el espacio $\left(S_{i}\right)=S_{i} \times s_{i}$ es el espacio producto $P_{i} \circ P_{i}$. Los elementos de $P_{i} \circ P_{i}$ se denotan $\mathrm{p}_{i}$.

4) Análogamente si s ordinarias a las cuales algún elemento de $\left.\mathrm{C}_{\left(\mathrm{P}_{i}\right.}\right)$ asigna probabilidad positiva, entonces $C\left(P_{i}^{\prime}\right) \circ C\left(P_{i}^{\prime}\right)$ será el conjunto de estrategias mixtas sobre el conjunto de estrategias normalizadas (s i), definidas como elementos del producto $\left(S_{i}^{\prime}\right)=S_{i}^{\prime} \times S_{i}^{\prime}$.

Así pues, el paso a la forma normal se reduce, simplemente, a calcular el pago esperado con respecto a la distribución $\mathrm{R}^{\star}$ sobre todo el espacio $C=C_{i} \times C_{2}$, es decir, a determinar para cada $(s)=\left(\left(s_{1}\right)\right.$, $\left.\left(s_{2}\right)\right)$ perteneciente al conjunto producto de los conjuntos de estrategias puras y ordinarias que son caracterizados por los correspondientes valores que asuma el vector $c=\left(c_{1}, c_{2}\right)$, cual es el valor que, a juicio del jugador 1 que se considera el analista en este caso, asume la expresión: 


$$
\begin{aligned}
& \mathrm{w}_{1}\left(\left(\mathrm{~s}_{1}\right),\left(\mathrm{s}_{2}\right)\right)=\mathrm{k}_{1}, \mathrm{k}_{2}=1,2 \quad \mathrm{U}_{1}\left(\left(\mathrm{~s}_{1}\right)\left(\mathrm{c}_{1}{ }^{1}\right),\left(\mathrm{s}_{2}\right)\left(\mathrm{c}_{2}{ }^{2}\right)\right) \quad \operatorname{Prob}\left(\mathrm{c}_{1}=\mathrm{c}_{1}{ }^{1}, \mathrm{c}_{2}=\mathrm{c}_{2}{ }^{2}\right) \\
& =u_{1}\left(\left(s_{1}\right)\left(c_{1}^{1}\right),\left(s_{2}\right)\left(c_{2}^{1}\right)\right)\left(1-t_{2}(1)\right)\left(1-r_{1}(2)\right) \\
& +u_{1}\left(\left(s_{1}\right)\left(c_{1}^{1}\right),\left(s_{2}\right)\left(c_{2}^{2}\right)\right)\left(1-t_{2}(1)\right) r_{1}(2) \\
& +U_{1}\left(\left(s_{1}\right)\left(c_{1}^{2}\right),\left(s_{2}\right)\left(c_{2}^{\prime}\right)\right)\left(t_{2}(1)\left(1-r_{i}(2)\right)\right. \\
& \left.+U_{1}\left(s_{1}\right)\left(c_{1}^{2}\right),\left(s_{2}\right)\left(c_{2}^{2}\right)\right) t_{2}(1) r_{1}(2) \\
& \mathrm{w}_{2}\left(\left(\mathrm{~s}_{1}\right),\left(\mathrm{s}_{2}\right)\right)=\mathrm{U}_{2}\left(\left(\mathrm{~s}_{1}\right)\left(\mathrm{c}_{1}^{1}\right),\left(\mathrm{s}_{2}\right)\left(\mathrm{c}_{2}^{\prime}\right)\right)\left(1-\mathrm{t}_{2}(1)\right)\left(1-\mathrm{r}_{1}(2)\right) \\
& \text { +...................... . + } \\
& U_{2}\left(\left(s_{1}\right)\left(c_{1}^{2}\right),\left(s_{2}\right)\left(c_{2}^{2}\right)\right) t_{2}(1) r_{1}(2)
\end{aligned}
$$

Expresiones análogas se obtienen si el analista fuera el jugador 2. En realidad, las expresiones diferirán solo en los últimos factores de cada expresión, en lugar de $r_{1}$ (2) y $t_{2}$ (1) figurarán $r_{2}$ (1) y $t_{1}(2)$.

Para comodidad notacional, se pueden escribir las estrategias normalizadas en orden lexicográfico, del modo siguiente:

si $e_{i}^{\text {ht }}$ es la estrategia normalizada correspondiente al jugador $i$, tal que al valor $c_{i}=c_{i}^{1}$ lde asigna la estrategia ordinaria $s_{i}^{h} \in s_{i}$, enton ces el lugar que ella ocupa es 1 , anotándose ahora $e_{i}^{1}$, en que $1=(h-1) k_{i}+t ; h, t=1, \ldots, k_{i}$

Así, con esta notación $\left(S_{1}\right)\left(c_{1}^{1}\right)$ se puede anotar también

$$
e_{1}^{\text {ht }}\left(c_{1}^{1}\right)=\left(s_{1}^{h}\right) ; e_{1}^{\text {ht }}\left(c_{1}^{2}\right)=s_{1}^{t}
$$

o bien

$$
e_{1}^{h t}\left(c_{1}\right)=\left(s_{1}^{h}, s_{1}^{t}\right)
$$


Ciertamente, la estrategia $\left(s_{1}\right)$ queda en esta nueva notación caracterizada por el par $(h, t)$, y su ubicación en el ordenamiento establecido, queda definido por la fórmula $1=(h-1) k_{i}+t$. Conviene observar que la función definida sobre el producto $K_{i} \times K_{i}$ con valores en el conjunto de los $k_{i}^{2}$ primeros enteros, por $f(h, t)=(h-1) K_{i}+t=1$ es una biyección, pues $h, t, 1$ son enteros $y$, por lo tanto,

$$
1=t \bmod k_{i}, h=\frac{1-t}{k_{i}}+1
$$

Por otro lado, es evidente que las estrategias puras ordinarias pertenecientes al conjunto $s_{i}$, pueden ser reordenadas de modo que las primeras $K_{i}^{\prime}$ sean, precisamente, las estrategias del conjunto $S_{i}^{\prime}$. De esta forma, el ordenamiento de las estrategias normalizadas permite identificarlas en sus respectivos conjuntos productos. Así pues:

$$
\begin{aligned}
& e_{i}^{1} \in s_{i}^{\prime} \times s_{i}^{\prime} \Longleftrightarrow 1 \leqslant h \leqslant k_{i}^{\prime}, 1 \leqslant t \leqslant k_{i}^{\prime} \\
& e_{i}^{1} \in s_{i}^{\prime} \times s_{i} \Longleftrightarrow 1 \leqslant h \leqslant k_{i}^{\prime}, 1 \leqslant t \leqslant k_{i} \\
& e_{i}^{1} \in s_{i} \times s_{i} \Longleftrightarrow 1 \leqslant h \leqslant k_{i} ; 1 \leqslant t \leqslant k_{i} \\
& e_{i}^{1} \in s_{i} \times s_{i}^{\prime} \Longleftrightarrow 1 \leqslant h \leqslant k_{i} ; 1 \leqslant t \leqslant k_{i}^{\prime}
\end{aligned}
$$

Análogamente para el jugador 2 .

Finalmente, sintetizando las ideas anteriores, la forma normal adquiere la siguiente estructura:

$$
N(G)=\left\{\left\{e_{1}^{1}\right\} \sum_{1=1}^{2},\left\{e_{2}^{1}\right\} \mathrm{K}_{1=1}^{2} ; \mathrm{W}_{1}, \mathrm{w}_{2}\right\}
$$




\subsection{Solución de la forma normal.}

De acuerdo a las hipótesis y condiciones iniciales del problema, la solución por la vía de la forma normal puede abordarse con dos criterios. El punto de partida común es la existencia de puntos de equilibrio, de acuerdo al teorema de Nash, en estrategias mixtas. El primer criterio consiste en separar los cuatro juegos que caracterizan los valores posibles del vector $c=\left(c_{1}, c_{2}\right)$. Esto es, analizar por separado los siguientes juegos en forma normal:

$$
\begin{aligned}
& \mathrm{N}_{1}(G)=\left\{\left\{e_{1}^{1}: e_{1}^{1} e S_{1} x_{1}\right\},\left\{e_{2}^{1} e s_{2} x_{2}\right\} ; w_{1}, w_{2}\right\} \\
& N_{2}(G)=\left\{\left\{e_{1}^{1}: e_{1}^{1} e S_{1} x_{1}\right\},\left\{e_{2}^{1} e S_{2}^{\prime} x S_{2}^{\prime}\right\} ; w_{1}, w_{2}\right\} \\
& N_{3}(G)=\left\{\left\{e_{1}^{1}: e_{1}^{1} \in S_{1}^{\prime} x S_{1}^{\prime}\right\},\left\{e_{2}^{1} \in S_{2} x_{2}\right\} ; w_{1}, w_{2}\right\} \\
& N_{4}(G)=\left\{\left\{e_{1}^{1}: e_{1}^{1} e S_{1}^{\prime} x S_{1}^{\prime}\right\},\left\{e_{2}^{1} e S_{2}^{\prime} x S_{2}^{\prime}\right\} ; w_{1}, w_{2}\right\}
\end{aligned}
$$

El segundo criterio consiste en analizar el mismo juego $\mathrm{N}(\mathrm{G})=$ $N_{1}(G)$, en los cuatro contextos indicados a la luz del comportamiento de las estrategias mixtas. Así, los distintos casos surgen cuando las estrategias mixtas se escogen por los contrincantes de la siguiente forma:

Caso 1: $\quad \mathrm{p}_{1} \in \mathrm{P}_{1} \circ \mathrm{P}_{1} ; \mathrm{p}_{2} \in \mathrm{P}_{2} \circ \mathrm{P}_{2}$

Caso 2: $p_{1} \in p_{1}^{o p_{1}} ; p_{2}$ como la inyección de $p_{2}^{\prime} \in C\left(P_{2}^{\prime}\right) \circ C\left(P_{2}^{\prime}\right)$

$$
\text { en } \mathrm{P}_{2} \mathrm{OP}_{2} \text {. }
$$

Caso 3: $\quad p_{1}$ como la inyección de $p_{1}^{\prime} \in C\left(P_{1}^{\prime}\right) \circ C\left(P_{1}^{\prime}\right)$ en $P_{1} \circ P_{1} ; p_{2} \in P_{2} \circ P_{2}$.

Caso 4: $\quad p_{1}$ como la inyección de $p_{1}^{\prime} \in C\left(P_{1}^{\prime}\right) \circ C\left(P_{1}^{\prime}\right)$ en $P_{1} \circ P_{1} ; p_{2}$ como la inyección de $\mathrm{p}_{2}^{\prime} \in \mathrm{C}\left(\mathrm{P}_{2}^{\prime}\right) \propto \mathrm{C}\left(\mathrm{P}_{2}^{\prime}\right)$ en $\mathrm{P}_{2}{ }^{\circ} \mathrm{P}_{2}$. 
Esquemáticamente, esta última versión aparece como se indica en el cuadro siguiente para el caso particular dado por:

$$
\begin{array}{ll}
\mathrm{K}_{1}=5 & \mathrm{~K}_{1}^{\prime}=3 \\
\mathrm{~K}_{2}=4 & \mathrm{~K}_{2}^{\prime}=2
\end{array}
$$

En este cuadro, las estrategias normalizadas se identifican con su ordinal respectivo. Los ordinales encerrados en un círculo, corres ponden a las estrategias normalizadas correspondientes a las pertenecien tes a los conjuntos

$$
s_{i}^{\prime} \times s_{i}^{\prime} \quad(i=1,2)
$$

Los ceros que figuran en las filas y columnas marginales, corresponden a las componentes de las estrategias mixtas inyectadas en $P_{i}{ }^{O P}{ }_{i}$ Finalmente, los pares de valores correspondientes a las funciones $w_{1}$ y $w_{2}$ figuran representados simbólicamente, en forma del elemento genêrico de la bimatriz de pago. 


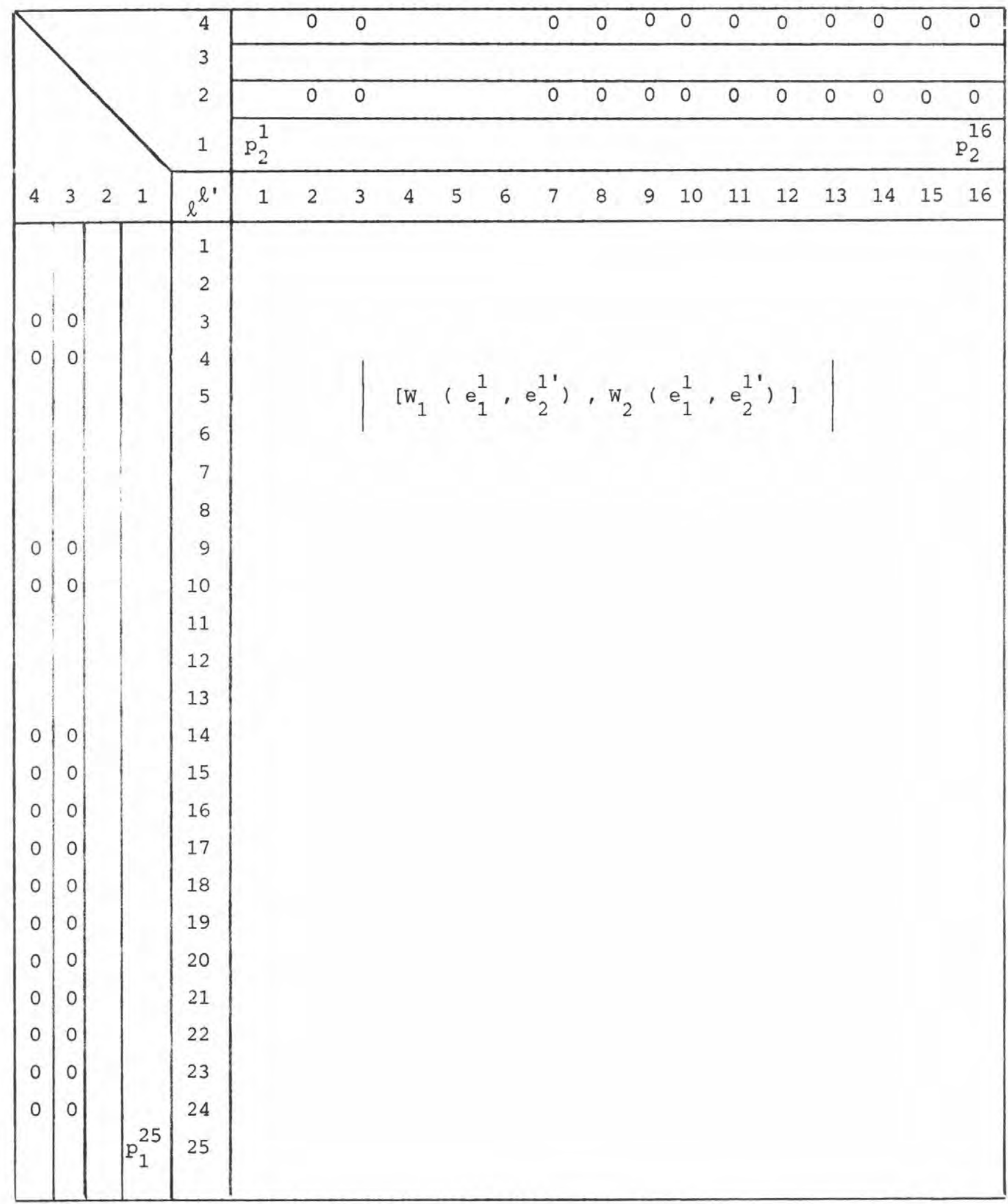


Es claro, que ambos criterios son equivalentes en términos de soluciones en el sentido de Nash. La última expresión facilita la visión de conjunto, mientras que la primera facilita la interpretación teórica de las soluciones. Tal como se ha señalado, el concepto de solución depende del caso que se analice. Así pues, se tendrá:

Caso 1: Ambos jugadores, en opinión del jugador 1 que es el analista, buscan el punto de equilibrio en estrategias mixtas. La existencia la garantiza, como ya se ha citado, el teorema de Nash. Los espacios de estrategias son $p_{1} \circ p_{1}$ para el jugador 1 y $p_{2}$ op 2 para el jugador 2 . Así pues, si

$$
\mathrm{p}_{1}^{*} \in \mathrm{P}_{1} \circ \mathrm{P}_{1}
$$

es la primera coordenada del punto de equilibrio y

$$
\stackrel{*}{\mathrm{p}}_{2} \in \mathrm{P}_{2} \circ \mathrm{P}_{2}
$$

es la segunda, se tendrán las siguientes afirmaciones:

$1^{\circ}$ ) Por definición de punto de equilibrio, $\stackrel{*}{p}_{1}$ es la "mejor respuesta" de 1 a la estrategia mixta $\stackrel{*}{p}_{2}$ que opone su contrario y reciprocamente.

$2^{\circ}$ ) El valor de información, es cero para ambos jugadores. Efectivamente, por definición

$$
\begin{aligned}
& I\left(\tilde{P}_{1}\right)=-\sum_{1,1}, w_{1}\left(e_{1}^{1}, e_{2}^{1}\right)\left(\stackrel{p}{1}_{1}^{1}\right)\left(\stackrel{p}{1}_{1}^{\prime}\right)+ \\
& \max _{p_{1} e_{1}^{2}} \sum_{1,1} w_{1}\left(e_{1}^{1}, e_{2}^{1 \prime}\right)\left(p_{1}^{1}\right)\left(*_{2}^{\prime}\right) \text { donde } \\
& \stackrel{\star}{\mathrm{p}}_{2}=\left({\stackrel{*}{p_{1}}}_{2}, \ldots,{\stackrel{*}{p_{1}}}_{2}, \ldots,{\stackrel{*}{\mathrm{p}_{2}}}_{2}^{2}\right) \text { es la segunda componente del }
\end{aligned}
$$

punto de equilibrio y por eso maximiza la expresión

$$
\sum_{1,1}, w_{2}\left(e_{1}^{1}, e_{2}^{1}\right)\left(\stackrel{p}{1}_{1}^{*}\right)\left(p_{2}^{1}\right) .
$$


Ahora si $p_{1}^{0}=\left(p_{1}^{0}, \ldots, p_{1}^{p_{1}}\right) \in p_{1}^{2}$ maximiza

$$
\sum w_{1}\left(e_{1}^{1}, e_{2}^{1}\right)\left(p_{1}^{1}\right)\left(\stackrel{*}{p}_{2}\right)
$$

se tendrá que:

$$
\sum_{1,1^{\prime}} w_{1}\left(e_{1}^{1}, e_{1}^{1}\right)\left(p_{1}^{1}\right)\left({\stackrel{p}{1} 1^{\prime}}_{2}\right) \geqslant \sum_{1,1^{\prime}} w_{1}\left(e_{1}^{1}, e_{2}^{1^{\prime}}\right)\left(\stackrel{*}{1}_{1}^{*}\right)\left(p_{2}^{*}\right)
$$

pero, ciertamente, es válida solo la igualdad de acuerdo a la definición de punto de equilibrio, es decir $\stackrel{0}{p}_{1}$ y $\stackrel{*}{p}_{1}$ son distribuciones "equivalen tes" en pago.

Analogamente se tiene:

$\sum, w_{2}\left(e_{1}^{1}, e_{2}^{1}\right)\left(\stackrel{p}{1}_{1}^{*}\right)\left(p_{2}^{1}\right) \geqslant \sum w_{2}\left(e_{1}^{1}, e_{2}^{1^{\prime}}\right)\left(*_{1}^{1}\right)\left(*_{2}^{\prime}\right)$ implica 1,1

que $\stackrel{0}{\mathrm{p}}$ es equivalente en pago a $\stackrel{*}{\mathrm{p}}_{2}$. Así pues, en definitiva, en opinión del analista 1 se cumple que:

$$
\sum_{1,1^{\prime}} w_{i}\left(e_{1}^{1}, e_{2}^{1}\right)\left(\stackrel{*}{p}_{1}^{*}\right)\left({\stackrel{*}{p_{2}^{\prime}}}^{\prime}\right)=\sum_{1,1} w_{i}\left(e_{1}^{1}, e_{2}^{1}\right)\left(p_{1}^{1}\right)\left(p_{2}^{1}\right)
$$

Es decir $\quad I\left(\tilde{P_{i}}\right)=0 \quad(i=1,2)$

$\left.3^{\circ}\right)$ Además, el punto de equilibrio $\stackrel{*}{\mathrm{p}}=\left(\stackrel{*}{\mathrm{p}_{1}},{\stackrel{*}{\mathrm{p}_{2}}}_{2}\right)$ induce en los respectivos espacios $\mathrm{P}_{1}$ y $\mathrm{P}_{2}$ distribuciones de probabilidad $\mathrm{q}_{1} \in \mathrm{P}_{i}$, ${\stackrel{\star}{q_{2}}}_{2}$ e $\mathrm{P}_{2}$ tales que $\stackrel{*}{q}=\left(\stackrel{*}{q}_{1}, \stackrel{*}{q}_{2}\right)$ es un punto de equilibrio para el juego $G^{*}=\left(S_{1}, S_{2} ; U_{1}, U_{2}\right)$, original.

La demostración es como sigue:

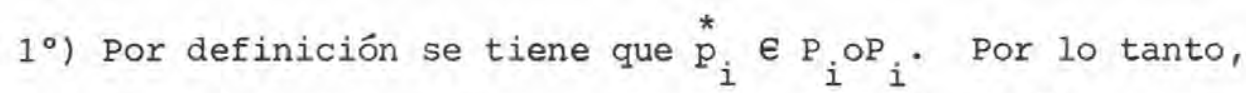

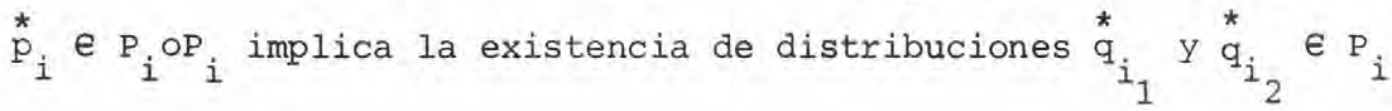


112

tal que:

$$
\text { si } \stackrel{*}{p}_{i}=\left(\stackrel{*}{p}_{i}^{1}, \stackrel{*}{p}_{1}^{2}, \ldots, \stackrel{*}{p}_{i}, \ldots, p_{i}^{k_{i}^{2}}\right)
$$

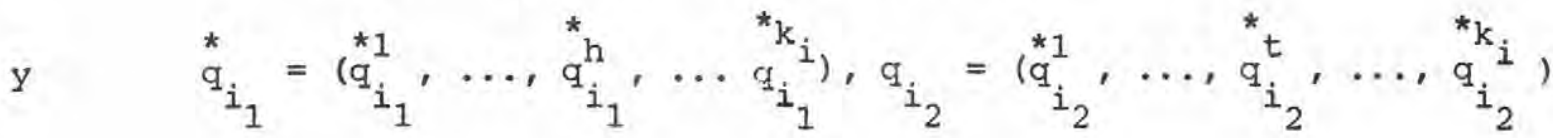

$$
\begin{aligned}
& \text { entonces } \stackrel{\star}{p}_{1}^{1}=q_{i_{1}}^{h} \quad q_{i_{2}}^{t} \text { con } 1=(h-1) k_{i}+t \text {. }
\end{aligned}
$$

La explicación es obvia, pues corresponde a la interpretación de la estrategia normalizada $e_{i}^{1}=e_{i}^{h t}$.

$\left.2^{\circ}\right)$ Así, la expresión $\sum_{1,1} w_{i}\left(e_{1}^{1}, e_{2}^{1}\right)\left(\stackrel{*}{p}_{1}^{1}\right)\left(p_{2}^{1}\right)$, aplicanco la definición de $w_{i} y$ el hecho de $\operatorname{ser} c_{1}=c_{1}^{1}$ y $c_{2}=c_{2}^{1}$, adquiere la forma:

$$
\begin{aligned}
& \sum_{1,1^{\prime}} w_{i}\left(e_{1}^{1}, e_{2}^{1}\right)\left(p_{1}^{*}\right)\left(\stackrel{*}{1}_{2}^{\prime}\right)=\sum_{1,1}\left[\sum U _ { i } ( s _ { 1 } ^ { h } , s _ { 2 } ^ { h ^ { \prime } } ) \left(1-r_{1}(2)\left(1-t_{2}(1)\right]\left(p_{1}^{1}\right)\left(p_{2}^{* 1}\right)\right.\right.
\end{aligned}
$$

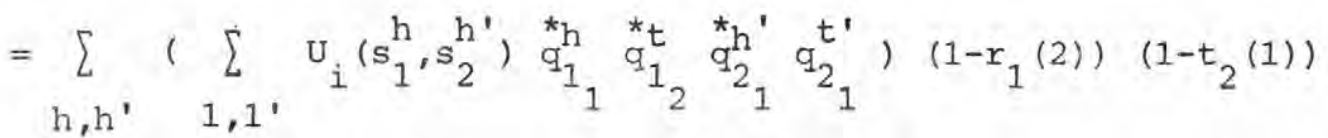

$$
\begin{aligned}
& =\left(\sum_{h, h^{\prime}} U_{i}\left(s_{1}^{h}, s_{2}^{h^{\prime}}\right){ }^{* h} q_{1}^{* h^{\prime}} q_{2}^{\prime}\right)\left(1-r_{1}(2)\left(1-t_{2}(1)\right)\right. \\
& \text { pues } \sum_{1,1^{\prime}}\left[{\stackrel{*}{p_{1}}{ }_{1}^{*} p_{2}^{\prime}}^{\prime}=\sum_{h, h^{\prime}} \sum_{t, t^{\prime}}[] q_{1_{1}}^{h} q_{2}^{h^{\prime}} q_{1}^{* t} q_{2}^{*} t_{2}^{\prime}\right.
\end{aligned}
$$

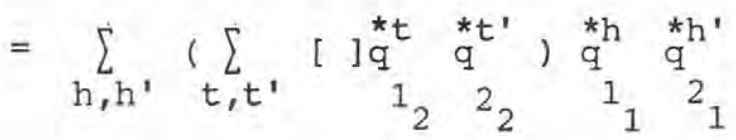

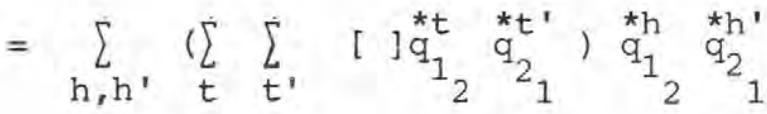




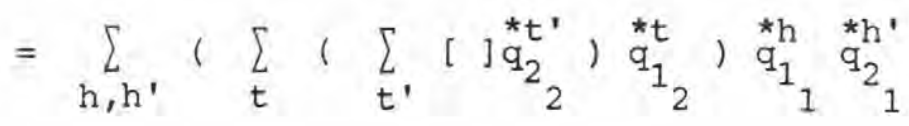

$$
\begin{aligned}
& =\sum_{h, h^{\prime}}\left(\sum_{t}[]_{1_{1}}^{* t}\right){\stackrel{* h}{q_{1}}}_{q_{1}}^{* h_{2}^{\prime}} \\
& =\sum_{h, h^{\prime}}[]^{* h} q_{1}^{* h^{\prime}}{ }_{2}^{\prime}
\end{aligned}
$$

Ciertamente, el paréntesis [ ] no depende de $t$ ni de $t^{\prime} y$, además

$$
\sum_{t}{ }^{* t}{ }_{12}^{*}=1 \quad \text { y } \quad \sum_{t^{\prime}} \quad{ }^{* t_{2}^{\prime}}=1 .
$$

Por lo tanto, si la expresión de la izquierda es el valor esperado de la función $\mathrm{w}_{i}$ para el punto de equilibrio, la expresión de la de recha será el pago esperado de la función $U_{i}$ para su correspondiente pun to de equilibrio.

Caso 2: El jugador 1, que es el analista, se pone en la posición de un jugador conservador $\left(c_{1}=c_{1}^{1}\right)$ y asigna a su contrincante una actitud agresiva $\left(c_{2}=c_{2}^{2}\right)$. En tal caso, son válidas las siguientes afirmaciones:

$\left.1^{\circ}\right)$ El jugador 1 emplea la estrategia mixta definida por el punto de equilibrio $\stackrel{*}{p}=\left(\stackrel{*}{p}_{1}, \stackrel{*}{p}_{2}\right)$.

$\left.2^{\circ}\right)$ El jugador 2 emplea la estrategia mixta definida por el máximo que alcanza el valor esperado de $\mathrm{W}_{2}$ sobre el espacio $\widetilde{\mathrm{P}}_{2}=\left\{\begin{array}{l}\mathrm{p}_{1} \\ \mathrm{~N}\end{array} \mathrm{x} \mathrm{C}^{2}\left(\mathrm{P}_{2}^{\prime}\right)\right.$. Sea $\tilde{p}(2)=\left(\tilde{p}_{1},{\stackrel{0}{p_{2}}}_{2}\right)$ uno de los elementos de $\tilde{\mathrm{p}}_{2}$ donde ocurre el máximo de $w_{2}$. Entonces el valor de información para el analista, jugador 1 , será en este caso:

$$
\begin{aligned}
& I\left(\mathrm{P}_{1}\right)=-\sum_{1,1,{ }^{\prime}} \mathrm{w}_{1}\left(\mathrm{e}_{1}^{1}, \mathrm{e}_{2}^{1}\right)\left(\stackrel{\mathrm{p}}{1}_{1}\right)\left(\stackrel{\mathrm{p}}{1}_{2}^{\prime}\right)+
\end{aligned}
$$

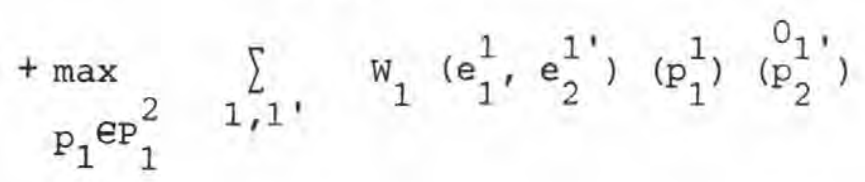


Ciertamente, para conservar el mismo recorrido de los superíndi ces, en lugar de considerar a $\mathrm{P}_{2}^{\prime}$ como elemento de $C^{2}\left(\mathrm{P}_{2}^{\prime}\right)$ se considera su inyección en $\mathrm{P}_{2} \circ \mathrm{P}_{2}=\mathrm{P}_{2}^{2}$

$\left.3^{\circ}\right)$ Análogamente al caso anterior, las distribuciones que efectivamente emplean los contrincantes, inducen en el juego original, distribuciones - estrategias mixtas cuyos significados son los mismos que tienen las es trategias mixtas normalizadas que las originan.

Así ${\stackrel{0}{p_{1}}}_{1}$, que maximiza $\left[\mathrm{W}_{1}\left(\mathrm{e}_{1}^{1}, \mathrm{e}_{2}^{1}\right)\left(\mathrm{p}_{1}^{1}\right)\left(\mathrm{p}_{2}^{1}\right)\right.$, induce la distribución $\stackrel{0}{q}_{1}$ e $\mathrm{P}_{1}, \mathrm{y}$ la distribución ${\stackrel{0}{\mathrm{p}_{2}}}_{2}$ que maximiza:

$\sum \mathrm{W}_{2}\left(\mathrm{e}^{1}, \mathrm{e}^{1}\right)\left(\stackrel{\mathrm{p}_{1}^{1}}{\mathrm{p}}\right)\left(\stackrel{\mathrm{p}_{2}^{1}}{\mathrm{p}^{\prime}}\right)$ induce la distribución $\stackrel{\mathrm{q}}{\mathrm{q}}_{2} \in \mathrm{P}_{2}$. Además del pun to anterior se sabe que $\stackrel{*}{\mathrm{p}}_{1} \in \mathrm{P}_{1} \circ \mathrm{P}_{1}$ induce $\stackrel{*}{\mathrm{q}}_{1} \in \mathrm{P}_{1}$ que es la primera coor denada del punto de equilibrio $\stackrel{*}{q}=\left(\stackrel{*}{q}_{1}, \stackrel{*}{q_{2}}\right)$ del juego:

$$
G=\left(S_{1}, S_{2} ; U_{1}, U_{2}\right) \text {. }
$$

Con estas notaciones, la demostración de la consistencia entre ambos modelos es análoga a la del punto anterior. En este caso $c_{1}=c_{1}^{1}$ implica que las estrategias puras ordinarias, que determinan la estrate gia mixta $\mathrm{p}_{1}$ son las correspondientes a los valores de $h$, tales que $\mathrm{p}_{1}=\stackrel{\mathrm{O}}{\mathrm{q}}_{1}^{\mathrm{O}} \mathrm{q}_{1_{2}}^{\mathrm{q}_{\mathrm{t}}}$ asume valores positivos. Análogamente, las estrategias pu ras ordinarias correspondientes al jugador 2, quedan definidas por los valores de $t$ para los cuales $p_{2}=q_{2} q_{1} q_{2}$ es positivo, pues el caso que se analiza corresponde a $c_{2}=c_{2}^{2}$. 
Caso 3: Ahora, el analista se pone en la situación de un jugador agresivo $\left(c_{1}=c_{1}^{2}\right)$ y asigna a su contrincante una actitud conservadora $\left(c_{2}=c_{2}^{1}\right)$. En estas condiciones serán válidas las siguientes afirmacio nes:

1\%) El jugador 1 emplea la estrategia que maximiza la expresión

$$
\sum w_{1}\left(e_{1}^{1}, e_{2}^{1 '}\right)\left(p_{1}^{1}\right)\left(p_{2}^{\star}{ }^{\prime}\right)
$$

en que ${\stackrel{*}{p_{2}}}_{2}$ es la segunda coordenada del punto de equilibrio $\stackrel{*}{\mathrm{p}}=\left(\stackrel{*}{\mathrm{p}}_{1}, \stackrel{*}{\mathrm{p}_{2}}\right)$.

$2^{\circ}$ ) El jugador emplea la estrategia mixta dada por el punto de equili brio.

En estas condiciones, el valor de información queda definido por la siguiente expresión:

$$
I(\tilde{P})=-\sum w_{1}\left(e_{1}^{1}, e_{2}^{1}\right)\left(\tilde{p}_{1}^{*}\right)\left(\tilde{p}_{2}^{\prime}\right)+\max _{\operatorname{pec}^{2}\left(p_{1}^{\prime}\right)} \sum w_{1}\left(e_{1}^{1}, e_{2}^{1}\right)\left(p_{1}^{1}\right)\left(\tilde{p}_{2}^{\prime}\right)
$$

Caso 4: Finalmente, el analista analiza la situación desde una perspec tiva $\left(c_{1}=c_{1}^{2}\right)$ para enfrentarse a un contrincante también agresivo $\left(c_{2}=c_{2}^{2}\right)$. Los comportamientos respectivos serán ahora, como se indica.

$1^{\circ}$ ) El jugador 1 , el analista, decide aplicar la estrategia mixta que maximiza en $\mathrm{p}_{1}^{\prime} \in \mathrm{C}^{2}\left(\mathrm{P}_{1}^{\prime}\right)$ la expresión

$$
\sum_{1,1}, w_{1}\left(e_{1}^{1}, e_{2}^{1 '}\right)\left(p_{1}^{\prime 1}\right)\left(p_{2}^{\prime} 1^{\prime}\right)
$$

donde ${\stackrel{0}{p_{1}}}_{1}$ es la estrategia mixta del jugador 2 que maximiza la expresión

$$
\begin{array}{r}
\sum_{1,1^{\prime}} w_{2}\left(e_{1}^{1}, e_{2}^{I^{\prime}}\right)\left(p_{1}^{\prime l}\right)\left(p_{2}^{\prime}{ }^{\prime}\right) \text { para } p_{2}^{\prime} \in C^{2}\left(P_{2}^{\prime}\right), y \\
p_{1}^{\prime} \in C^{2}\left(P_{1}^{\prime}\right) .
\end{array}
$$


$2^{\circ}$ ) El jugador 2 aplica la estrategia mixta ${\stackrel{0}{p_{2}}}_{2}$ definida en el punto $1^{\circ}$ ).

Con estos criterios, el analista calcula el valor de la informa ción correspondiente, que en este caso resulta ser:

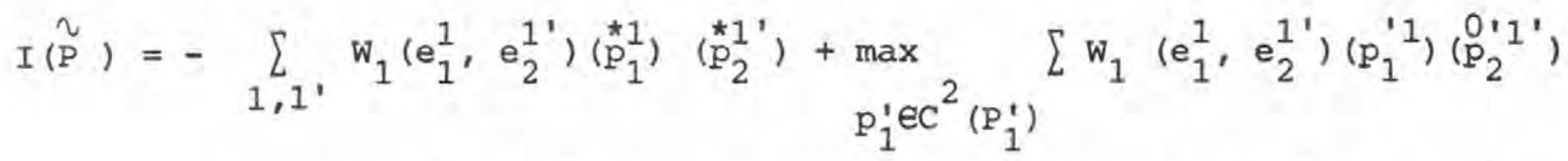

Nuevamente las estrategias mixtas normalizadas inducen $y$, con las mismas interpretaciones, las correspondientes estrategias mixtas ordinarias correspondientes al juego original $\mathrm{G}=\left(\mathrm{S}_{1}, \mathrm{~S}_{2} ; \mathrm{U}_{1}, \mathrm{U}_{2}\right)$.

\subsection{Reglas de decisión.}

A la luz del análisis anterior, el analista, en este caso el ju gador 1 , cuenta con dos elementos para decidir:

- el valor de información en cada caso

- la posibilidad con que esto ocurre.

Así pues, un criterio de racionalidad para el jugador 1, será: Si $\mathrm{H}_{\alpha}$ es el valor de información en los casos $\alpha=1,2,3,4$ y $t$ la posibilidad asignada a $\mathrm{H}_{\alpha}$, entonces el analista opta por la estrategia mixta, en la cual el producto $\mathrm{H}_{\alpha} t_{\alpha}$ sea máximo.

Nivel de Seguridad. Ciertamente que las situaciones favorables al jugador analista pueden ocurrir con probabilidad muy baja, por eso el analis ta puede fijarse un cierto nivel de confianza, tanto para su "credibilidad", como su estimación de la credibilidad que su oponente asigna a su caracterización. Ciertamente, esta actitud puede ser asumida por el ana lista como un dato inicial del problema.

Los diversos elementos que intervienen en la decisión del analista 1, se presentan en los siguientes cuadros: 


\begin{tabular}{|l|l|c|}
\hline \multicolumn{1}{|c|}{$c_{2}=c_{2}^{1}$} & $c_{\alpha}=c_{2}^{2}$ \\
\hline$c_{1}=c_{1}^{1}$ & {$\left[1-r_{1}(2)\right]\left[1-t_{2}(1)\right.$} & {$\left[1-r_{1}(2)\right] t_{2}(1)$} \\
\hline$c_{1}=c_{1}^{2}$ & $r_{1}(2)\left[1-t_{2}(1)\right]$ & $r_{1}(2) t_{2}(1)$ \\
\hline
\end{tabular}

\begin{tabular}{|l|c|c|} 
& $c_{2}=c_{2}^{\prime}{ }^{H_{\alpha}}$ & $c_{2}=c_{2}^{2}$ \\
\hline$c_{1}=c_{1}^{1}$ & $H_{1}$ & $\mathrm{H}_{2}$ \\
\hline$c_{1}=c_{1}^{2}$ & $\mathrm{H}_{3}$ & $\mathrm{H}_{4}$ \\
\hline
\end{tabular}

Espacios de estrategias mixtas para los jugadores 1 y 2.

\begin{tabular}{|l|l|l|}
\multicolumn{1}{c|}{} & $\mathrm{C}_{2}=\mathrm{c}_{2}^{1}$ & \multicolumn{1}{c|}{$\mathrm{c}_{2}=\mathrm{c}_{2}^{2}$} \\
\hline $\mathrm{C}_{1}=\mathrm{c}_{1}^{1}$ & 1) $\mathrm{P}_{2}^{2}$ & 1) $\mathrm{P}_{1}^{2}$ \\
\hline $\mathrm{C}_{1}=\mathrm{c}_{1}^{2}$ & 1) $\mathrm{C}^{2}\left(\mathrm{P}_{1}^{\prime}\right)$ & 2) $\mathrm{C}^{2}\left(\mathrm{P}_{2}^{\prime}\right)$ \\
\hline & 2) $\mathrm{P}_{2}^{2}$ & 1) $\mathrm{C}^{2}\left(\mathrm{P}_{1}^{\prime}\right)$ \\
\hline
\end{tabular}


Ciertamente, el análisis del jugador 1 tiene como elemento personal la distribución de probabilidad sobre el espacio $c=c_{1} \times c_{2}=\left\{\left(c_{1}^{1}, c_{2}^{\prime}\right),\left(c_{1}^{1}, c_{2}^{2}\right),\left(c_{1}^{2}, c_{2}^{\prime}\right),\left(c_{1}^{2}, c_{2}^{2}\right)\right\}$. Para él, esta distribución satisface la siguientes condiciones:

$\left.1^{\circ}\right)$ Las variables $c_{1} y_{2} c_{2}$ son estocásticamente independientes $y$, por 10 tanto, las distribuciones marginales con respecto a la distribución conjunta $R_{1}^{*}\left(1, c_{2}\right)$ coinciden con las distribuciones condicionales $R_{1}^{*}\left(c_{2} \mid c_{1}\right) \circ R_{2}^{*}\left(c_{1} \mid c_{2}\right)$ según corresponda.

$\left.2^{\circ}\right)$ El comportamiento del analista y de su contrincante es bayesiano. Así, las distribuciones condicionales $R_{1}\left(c_{2} \mid c_{1}\right)$ y $R_{2}\left(c_{1} \mid c_{2}\right)$ serán de naturaleza subjetiva pero, que en términos del analista satisfacen las relaciones:

$R_{1}^{*}\left(c_{2} \mid c_{1}\right)=R_{1} \quad\left(c_{2} \mid c_{1}\right)$ y $R_{2}^{*}\left(c_{1} \mid c_{2}\right)=R_{2} \quad\left(c_{1} \mid c_{2}\right)$

$\left.3^{\circ}\right)$ En opinión del analista, la distribución conjunta $R^{*}\left(c_{1}, c_{2}\right)$ satisface, además, los siguientes valores:

$$
\begin{aligned}
& R^{*}\left(c_{2}=c_{2}^{1}\right)=1-r_{1}(2) \\
& R^{*}\left(c_{2}=c_{2}^{2}\right)=r_{i}(2) \\
& R^{*}\left(c_{2}=c_{1}^{1}\right)=1-t_{2}(1) \\
& R^{*}\left(c_{2}=c_{1}^{2}\right)=t_{2}(1)
\end{aligned}
$$

Así, el análisis del jugador 1 tiene en último término como se110 personal, la probabilidad $r_{1}(2)$, que representa su confiabilidad $y$, la probabilidad $t_{2}(1)$ que representa la estimación que 1 formula de la confiabilidad del jugador 2. Por lo tanto, el análisis que haga el ju- 
gador 2, diferirá del que realice el jugador 1 solo en los valores que 2 asigne a $R^{*}\left(c_{1}, c_{2}\right)$, conservando los mismos criterios de los puntos $1^{\circ} y$ $2^{\circ}$. Ciertamente, si $r_{2}(1)=R^{*}\left(c_{1}, c_{1}^{2}\right)$ en opinión del jugador 2 , se podrá escribir que la estimación del valor $r_{2}(1)$ es en opinión de 1 el valor $t_{2}$ (1) y la estimación del valor $r_{1}(2)$ en opinión de 2 , será $t_{1}(2)$.

De estas consideraciones, surge como conclusión que el modelo hasta aquí presentado, tendrá tanta mayor validez cuanto más exactas sean las estimaciones $t_{2}(1)$ y $t_{1}(2)$, pues en tal caso, las distribucio nes $R^{*}$ que formulen por separado ambos jugadores, tenderán a coincidir.

Podría argumentarse que $\mathrm{R}^{*}$ es un dato conocido por ambos jugado res. Ciertamente, puede avalar esta afirmación el suponer que los valores que asume $R^{*}\left(c_{1}, c_{2}\right)$ tienen un origen experimental. No cabe duda, si los mismos contrincantes se han enfrentado en diversas ocasiones, el pro ceso de inferencia podría basarse en un análisis del tipo frecuentista.

Finalmente, se puede constatar que la aproximación bayesiana pro porciona una metodología racional para la adopción de decisiones para los juegos bipersonales, bimatriciales son "información no segura". La generalización para el caso de $n$ jugadores, admite un tratamiento entreramente similar, no existiendo a priori ninguna nueva hipótesis adicional.

\section{BIBLIOGRAFIA.}

[1] Harsanyi, John: "Games with Incomplete Information Played by Bayesian Players". Managament Science. Vol 14.

[2] Selten, Richard: "Reexamination of the Perfectness Concept for Equilibrium Points in Extensive Games". Inter. Journal of Game Theory Vol. 4. Issue 1.

[3] Prado, Arnoldo: "Enfoque Bayesiano para el valor de Información en Juegos". Tesis Doctoral. Universidad de Madrid. 